\title{
Communication
}

\section{Synthesis of a Highly Luminescent Three-Dimensional Pyrene Dye Based on the Spirobifluorene Skeleton}

\author{
Kentaro Sumi and Gen-ichi Konishi * \\ Department of Organic and Polymeric Materials, Graduate School of Science and Engineering, Tokyo \\ Institute of Technology, Japan
}

* Author to whom correspondence should be addressed; E-mail: konishi.g.aa@m.titech.ac.jp.

Received: 24 August 2010; in revised form: 25 October 2010 / Accepted: 27 October 2010 /

Published: 28 October 2010

\begin{abstract}
We have synthesized a highly luminescent $(\log \varepsilon>5.0, \Phi>0.9)$ pyrene dye based on a spirobifluorene skeleton [2,2',7,7'-tetrakis(7-tert-butyl-1-pyrenyl)-9,9'spirobi[9H-fluorene; 4-PySBF]. The use of spirobifluorene prevents fluorescence quenching by intramolecular energy transfer and/or electron transfer among the chromophores in the excited state. The emission spectra of 4-PySBF exhibited a red shift of $20 \mathrm{~nm}$ in comparison to a model compound [9,9'-dioctyl-2,7-bis(7-tert-butyl-1-pyrenyl)9H-fluorene; 2-PyF], but its UV-Vis spectrum remained unchanged.
\end{abstract}

Keywords: Spirobifluorene; pyrene; fluorescence; $\pi$-conjugation; dye

\section{Introduction}

Recently, blue emitting dyes with high efficiency $(\Phi \approx 1.0, \log \varepsilon>4.5)$ [1] have been attracting considerable attention because of their applicability to molecular electronic materials such as organic field effect transistors (OFETs) and organic light-emitting diodes (OLEDs). Many studies have been conducted on strategies for synthesizing a highly efficient dye. For example, rod- $[1,2]$ and star-shaped [3-6] hydrocarbons that expand $\pi$-conjugation to two dimensions can be used to control the emission color of a dye with high efficiency.

Spirobifluorene derivatives are considered the most promising candidates for organic optoelectronics [7-11]. The rigidity of spiro-compounds affords them high thermal stability. In addition, their solubility is higher than that of corresponding compounds without a spiro moiety, 
because their perpendicular conformations that are based on the spiro-linkage efficiently suppress intermolecular interactions between $\pi$-systems. Recently, several studies were conducted on introducing dyes into a molecule for $\pi$-conjugation in three dimensions. Examples of such studies include those on the introduction of dyes into the spirobifluorene skeleton [12-19]. Because of the high thermal stability and unique characteristics of the spirobifluorene skeleton, it has been adopted in various materials [20-22].

In this paper, we report the synthesis and characterization of a new pyrene dye that is based on the spirobifluorene skeleton. Pyrene derivatives are highly absorptive [23-26]; further, the introduction of pyrene substituents into spirobifluorene derivatives is expected to improve the fluorescence quantum yield and thermal stability of the derivatives because the substituents are highly emissive, bulky, and rigid.

\section{Results and Discussion}

A spirobifluorene dye, 2,2',7,7'-tetrakis(7-tert-butyl-1-pyrenyl)-9,9'-spirobi[9H-fluorene] (4PySBF), and a model compound, 9,9'-dioctyl-2,7-bis(7-tert-butyl-1-pyrenyl)-9H-fluorene (2-PyF), were synthesized in $37 \%$ and $24 \%$ yields, respectively, by palladium-catalyzed Suzuki-Miyaura coupling reactions (Scheme 1). The precursors, 7-tert-butylpyrene-1-boronic acid pinacol ester (1) and 1-bromo-7-tert-butylpyrene (3), were prepared from pyrene according to a previously reported method [27]. The chemical structures of 4-PySBF and 2-PyF were confirmed by ${ }^{1} \mathrm{H}-\mathrm{NMR},{ }^{13} \mathrm{C}-\mathrm{NMR}$, and FTIR spectroscopy. Each compound was found to be soluble in various organic solvents such as chloroform, tetrahydrofuran (THF), dichloromethane, and toluene. It should be noted that the tertbutyl group is necessary for the successful synthesis of 4-PySBF; we found that without tert-butyl groups, the solubility is quite poor, making purification and structural analysis by techniques such as ${ }^{1} \mathrm{H}-\mathrm{NMR}$ and ${ }^{13} \mathrm{C}-\mathrm{NMR}$ rather difficult.

Scheme 1. Synthesis of spirobifluorene dye and model compound.

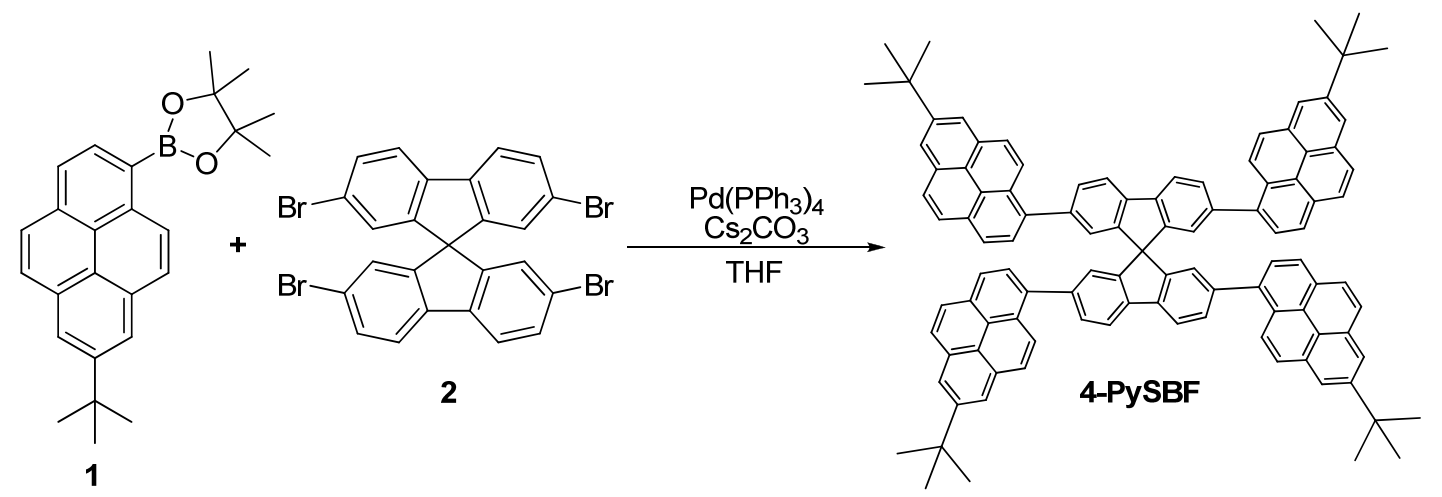<smiles>CC(C)(C)c1cc2ccc3ccc(Br)c4ccc(c1)c2c34</smiles>

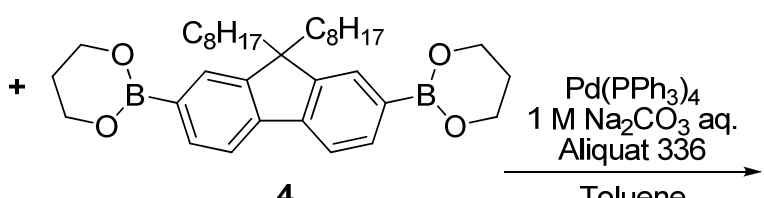

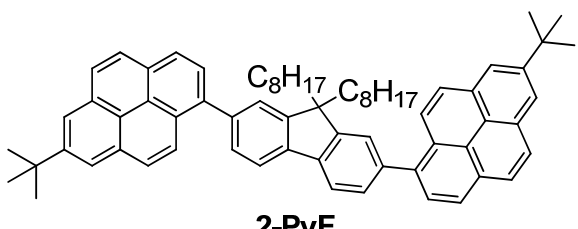


We measured the UV-Vis spectra, fluorescence spectra, fluorescence quantum yields, and fluorescence lifetimes, and lifetimes of 4-PySBF and 2-PyF in $\mathrm{CH}_{2} \mathrm{Cl}_{2}$ solution. The UV-Vis spectra (Figure 2, left side) show that the absorption maxima $\left(\lambda_{\max }\right)$ of 4-PySBF and 2-PyF are at $360 \mathrm{~nm}$ and $359 \mathrm{~nm}$, with molar absorption $\left(\lambda_{\max }\right)$ coefficients $\varepsilon$ of 131,000 and $71,000 \mathrm{M}^{-1} \mathrm{~cm}^{-1}$, respectively. The finding that the $\varepsilon$ value of 4-PySBF is about twice that of 2-PyF is in accordance with the fact that 4PySBF has two chromophores per molecule whereas 2-PyF has but one.

The observed fluorescence spectra (Figure 2, right side) show that 4-PySBF and 2-PyF exhibit intense blue emissions $\left(\lambda_{\mathrm{em}}\right)$ at $441 \mathrm{~nm}(\Phi=0.92)$ and $421 \mathrm{~nm}(\Phi=0.90)$, respectively, in $\mathrm{CH}_{2} \mathrm{Cl}_{2}$ solution $\left(\lambda_{\mathrm{ex}}=360 \mathrm{~nm} ; c=1.0 \times 10^{-6} \mathrm{M}\right)$. The fluorescence spectra also reveal that the fluorescence intensity of 4-PySBF is about twice that of 2-PyF, again because 4-PySBF has two chromophores per molecule. A 20-nm red-shift between 4-PySBF and 2-PyF was observed. It is noted that we observed a spiroconjugation (a large red shift in the fluorescence spectrum and no red shift in the absorption spectrum in this 4-PySBF and 2-PyF pair). We hypothesize that this observed red-shift could be due to conjugation of 4-PySBF via the spiro carbon [28-32] in the excited state [33]. Another possibility is a through-space interaction between upper and lower pyrene chromophores.

Figure 1. Absorption and emission spectra of 2-PyF and 4-PySBF in $\mathrm{CH}_{2} \mathrm{Cl}_{2} ; c=10 \mu \mathrm{M}$, $\lambda_{\mathrm{ex}}=360 \mathrm{~nm}$.

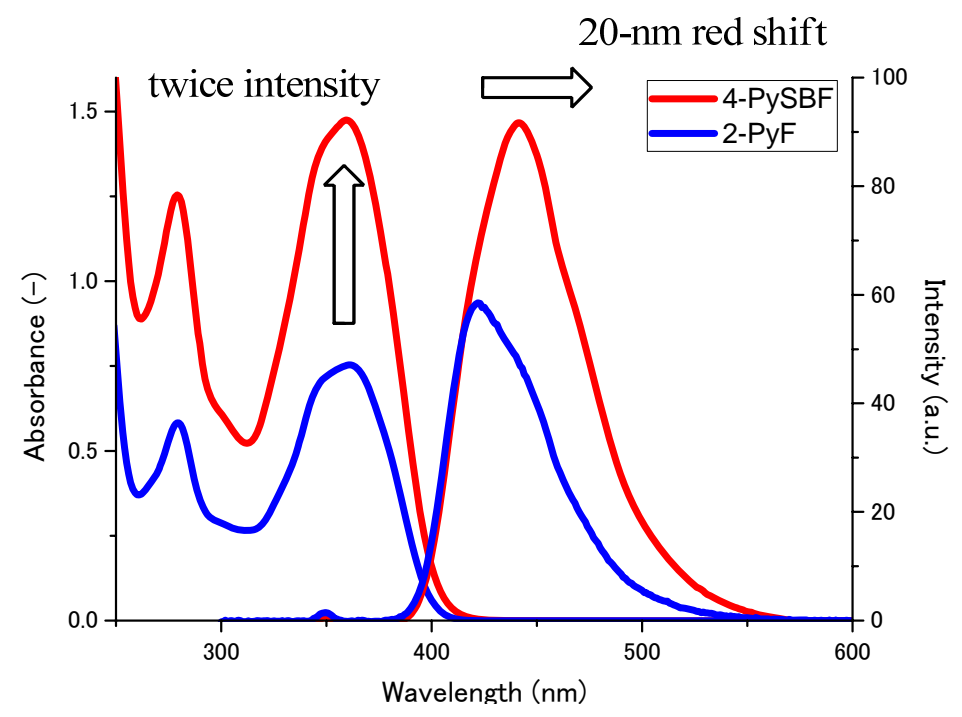

Table 1. Photochemical data for compounds 4-PySBF and 6.

\begin{tabular}{ccccccccc}
\hline dye & $\begin{array}{c}\lambda_{\text {ab }} \\
{[\mathbf{n m}]}\end{array}$ & $\begin{array}{c}\varepsilon\left(\times 10^{5}\right) \\
{[\mathbf{l} / \mathbf{m o l} \cdot \mathbf{c m}]}\end{array}$ & $\log \varepsilon$ & $\begin{array}{c}\lambda_{\text {em }} \\
{[\mathbf{n m}]}\end{array}$ & $\Phi$ & $\begin{array}{c}\tau \\
{[\mathbf{n s}]}\end{array}$ & $\begin{array}{c}\boldsymbol{k}_{\mathbf{f}}\left(\times \mathbf{1 0}^{\mathbf{8}}\right) \\
{\left[\mathbf{S}^{-1}\right]}\end{array}$ & $\begin{array}{c}\boldsymbol{k}_{\text {isc }}+\boldsymbol{k}_{\mathbf{n r}} \\
\left(\times \mathbf{1 0}^{\mathbf{7}}\right)\left[\mathbf{S}^{-\mathbf{1}}\right]\end{array}$ \\
\hline \hline 4-PySBF & 360 & 1.31 & 5.12 & 441 & 0.92 & 1.52 & 6.1 & 5.3 \\
2-PyF & 359 & 0.71 & 4.85 & 421 & 0.90 & 1.88 & 4.8 & 5.3 \\
\hline
\end{tabular}

Measurement of the photophysical properties of both compounds at the same concentration confirm that 4-PySBF had twice the number of chromophores in solution as compared to 2-PyF. Next, we measured the fluorescence spectra of both compounds under the same absorption conditions. We then found that the solutions of 4-PySBF and 2-PyF had the same number of chromophores. The fluorescence spectra (Figure 3) revealed that both 4-PySBF and 2-PyF had approximately the same 
fluorescence intensity. This result shows that the spirobifluorene derivatization doubled the performance of the chromophores by preventing intramolecular energy transfer and/or electron transfer, although the pyrene chromophores are placed close together. The compound 4-PySBF was found to have ideal luminescence properties $(\log \varepsilon>5.0, \Phi>0.9)$.

Figure 2. Emission spectra of 2-PyF and 4-PySBF in $\mathrm{CH}_{2} \mathrm{Cl}_{2} ; \mathrm{Abs}=0.1, \lambda_{\mathrm{ex}}=360 \mathrm{~nm}$.

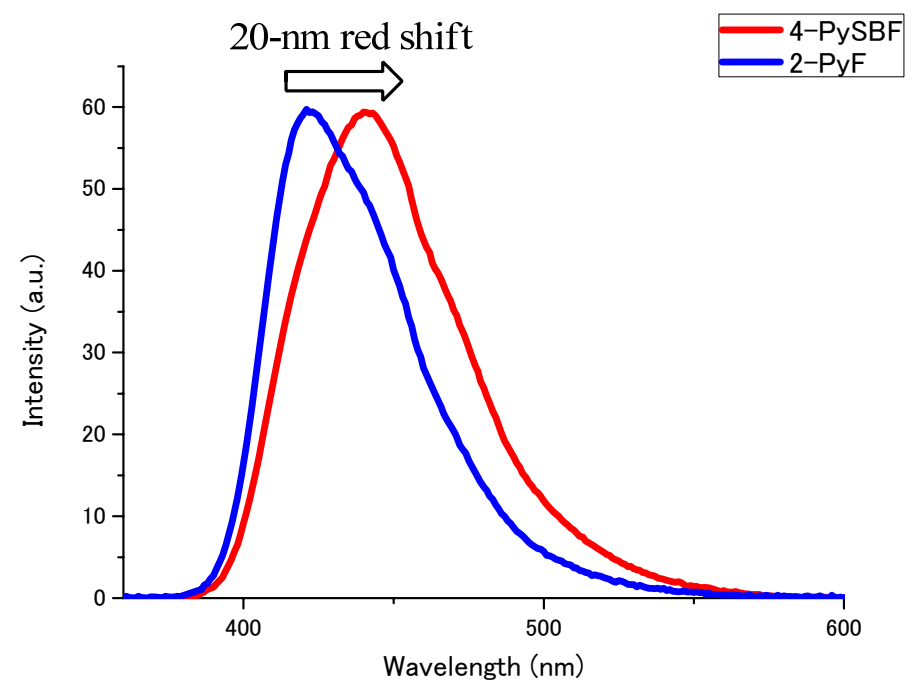

Fluorescence quantum yield can be expressed as $\Phi_{\mathrm{f}}=k_{\mathrm{f}} \tau_{\mathrm{s}}$, or $\Phi_{\mathrm{f}}=k_{\mathrm{f}} /\left(k_{\mathrm{f}}+k_{\mathrm{isc}}+k_{\mathrm{nr}}+k_{\mathrm{q}}\left[\mathrm{O}_{2}\right]\right)$, where $k_{\mathrm{f}}, k_{\mathrm{isc}}, k_{\mathrm{nr}}$, and $k_{\mathrm{q}}\left[\mathrm{O}_{2}\right]$ denote the rate constants for fluorescence radiation, intersystem crossing, nonradiative decay, and fluorescence quenching by oxygen, respectively, and $\tau_{\mathrm{s}}$ denotes the lifetime ( $\mathrm{s}=$ singlet) [14]. Table 1 summarizes the values of $k_{\mathrm{f}}$ and $k_{\text {isc }}+k_{\mathrm{nr}}$ for 4-PySBF and 2-PyF, calculated from the experimental values of $\Phi_{\mathrm{f}}$ and $\tau_{\mathrm{s}}$ under degassed conditions, that is, under the assumption that $k_{\mathrm{q}}\left[\mathrm{O}_{2}\right]=0$. As $k_{\mathrm{nr}}$ for aromatic hydrocarbons is known to be negligible [34], $k_{\text {isc }}$ should be the dominant component in $k_{\mathrm{isc}}+k_{\mathrm{nr}}$ [35-38]. Under aerated conditions, $k_{\mathrm{q}}\left[\mathrm{O}_{2}\right]$ is estimated to be nearly diffusion controlled (up to $3.89 \times 10^{10} \mathrm{~m}^{-1} \mathrm{~s}^{-1}$ in hexane) [34,39], and $\left[\mathrm{O}_{2}\right]$ is less than $2.0 \times 10^{-3} \mathrm{M}$ in usual solvents. These data imply that the maximum possible value of $k_{\mathrm{q}}\left[\mathrm{O}_{2}\right]$ is $8.0 \times 10^{7} \mathrm{~s}^{-1}$. As can be seen from Table 1 , the $k_{\mathrm{f}}$ values for 4-PySBF and 2-PyF are much larger than the $k_{\mathrm{q}}\left[\mathrm{O}_{2}\right]$ values. This situation is most probably responsible for the observed large values of $\Phi_{\mathrm{f}}$ for both compounds, even in the presence of oxygen.

\section{Conclusions}

In summary, we have synthesized a highly luminescent spirobifluorene dye $(\log \varepsilon>5.0, \Phi>0.9)$ with tetrapyrene chromophores (4-PySBF) and measured its photophysical properties. In comparison to a model compound, 2-PyF, the emission spectrum of 4-PySBF exhibited a red shift of $20 \mathrm{~nm}$, but its $\mathrm{UV}-\mathrm{Vis}$ spectrum remained unchanged. It is not easy to find a satisfactory explanation for this observed red-shift at the present stage. However, in our next study, we intend to calculate the electron states of these compounds in the ground and excited states by the molecular orbital (MO) method and examine this conjugation system in greater detail. Further, we intend to apply 4-PySBF in a previously developed cholesteric liquid crystal laser (distributed feedback laser) as the laser dye in the future. 


\section{Experimental}

\section{Instruments}

All the ${ }^{1} \mathrm{H}$ - and ${ }^{13} \mathrm{C}-\mathrm{NMR}$ spectra were recorded on a $400 \mathrm{MHz}$ JEOL LMN-EX400 instrument with tetramethylsilane (TMS) as the internal standard. FT-IR spectra were recorded on a JASCO FT-IR 469 plus spectrometer. Melting points were obtained by a Stuart Scientific Melting Point Apparatus SMP3. MS spectra (FAB) were obtained by JEOL JMS700 mass spectrometer. UV-Vis spectra were recorded with a Beckman Coulter DU800 UV-Vis Spectrophotometer. Fluorescence spectra were recorded on a JASCO FP-6500 Spectrofluorometer. Quantum Yields were measured by a Hamamatsu Photonics C9920-02 Absolute PL Quantum Yield Measurement system. Fluorescence lifetimes were measured using a Hamamatsu Photonics OB 920 Fluorescence Lifetime Spectrometer.

\section{Materials}

Unless otherwise noted, all reagents, chemicals and solvents were obtained from commercial sources and used without further purification. Pyrene, 2-chloro-2-methylpropane, $\operatorname{Pd}\left(\mathrm{PPh}_{3}\right)_{4}, 4,4,5,5-$ tetramethyl-1,3,2-dioxaborolane were obtained from TCI. 9,9-Dioctyl-fluorene-2,7-bis(trimethyleneborate) (4) and $\mathrm{PdCl}_{2}\left(\mathrm{PPh}_{3}\right)_{2}$, were obtained from Aldrich. 2,2',7,7'-Tetrabromospirobifluorene (2) was a gift of JFE Chemical Coporation (Japan).

\section{2,2',7,7'-tetrakis(7-tert-Butyl-1-pyrenyl)-9,9'-spirobi [9H-fluorene] (3, 4-PySBF)}

Under an argon atomosphere, 2,2',7,7'-tetrabromospirobifluorene $(0.25 \mathrm{~g}, 0.4 \mathrm{mmol})$, 7-tertbutylpyrene-1-boronic acid pinacol ester $(0.77 \mathrm{~g}, 2.0 \mathrm{mmol})$ and cesium carbonate $(5.21 \mathrm{~g}, 16 \mathrm{mmol})$ were mixed together with $\mathrm{Pd}\left(\mathrm{PPh}_{3}\right)_{4}(100 \mathrm{mg}, 0.1 \mathrm{mmol})$ and degassed THF $(30 \mathrm{~mL})$. The mixture was refluxed for $24 \mathrm{~h}$. After cooling to room temperature, the resulting mixture was extracted with chloroform. The organic extract was washed sequentially with water and brine and then dried over $\mathrm{MgSO}_{4}$. After removal of the solvent, the residue was purified by column chromatography (chloroform:hexane = 1:5) and preparative HPLC $\left(\mathrm{CHCl}_{3}\right)$. After removal of the solvent, the product was recrystallized from cyclohexane to afford 4-PySBF as a yellow powder in $37 \%$ yield. ${ }^{1} \mathrm{H}-\mathrm{NMR}$ (400 MHz, THF-d 8 ): $\delta$ 8.23-7.36 (m, Ar-H, 44H), 1.53 (s, tert-butyl-H, 36H) ppm.; ${ }^{13} \mathrm{C}-\mathrm{NMR}(100$ $\left.\mathrm{MHz}, \mathrm{CDCl}_{3}\right): \delta 149.3,149.1,140.9,140.7,137.4,131.3,130.7,130.5,130.3,128.4,128.2,128.1$, 127.7, 127.5, 127.3, 127.2, 126.5, 125.0, 124.9, 124.4, 123.1, 122.4, 122.0, 120.2 (aromatic C), 66.3 (spiro-C), $35.2\left(\mathrm{Ar}-\mathrm{C}\left(\mathrm{CH}_{3}\right)_{3}\right), 31.9\left(\mathrm{Ar}-\mathrm{C}\left(\mathrm{CH}_{3}\right)_{3}\right)$ ppm; FT-IR $(\mathrm{KBr})$ : 3042, 2961, 2901, 2867, 1594, 1457, $1227 \mathrm{~cm}^{-1}$; mp 286-288 ${ }^{\circ} \mathrm{C}$; HR-MS $\left(\mathrm{FAB}^{+}\right)$Calcd. for $\mathrm{C}_{105} \mathrm{H}_{80}$ [M] 1341.6294, found [M ${ }^{+}$] 1341.6290 .

\section{9,9'-Dioctyl-2,7-bis (7-tert-butyl-1-pyrenyl)-9H-fluorene (6, 2-PyF)}

Under an argon atomosphere, 2-bromo-7-tert-butylpyrene (0.8 g, $2.4 \mathrm{mmol})$ and 9,9-dioctyl-

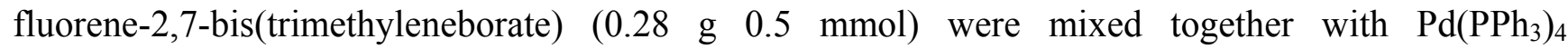
(60 mg, $0.05 \mathrm{mmol})$, degassed toluene $(20 \mathrm{~mL})$, one drop of Aliquat 336, and $1 \mathrm{M}$ aqueous sodium carbonate solution $(2 \mathrm{~mL})$. The resulting mixture was refluxed for $24 \mathrm{~h}$. After cooling to room 
temperature, the mixture was extracted with chloroform. The organic extract was washed sequentially with water and brine and then dried over $\mathrm{MgSO}_{4}$. After removal of the solvent, the residue was purified by column chromatography using chloroform/hexane $(5: 1, \mathrm{v} / \mathrm{v})$ following HPLC $\left(\mathrm{CHCl}_{3}\right)$. After subsequent recrystallization in ethanol, 2-PyF was obtained as a yellow powder in $24 \%$ yield. ${ }^{1} \mathrm{H}-\mathrm{NMR}\left(400 \mathrm{MHz}, \mathrm{CDCl}_{3}\right.$ ): 8 8.27-7.97 (m, Ar-H, 18H), 7.69-7.67 (m, Ar-H, 4H), 2.12-2.08 (s, ArCH-Ar, 2H), 1.60 (s, tert-butyl-H, 18H), 1.25-1.19 (m, alkyl-H, 20H), 0.96 (m, alkyl-H, 4H), 0.82 (t, $J=6.84$, alkyl-H, 6H) ppm; ${ }^{13} \mathrm{C}-\mathrm{NMR}\left(100 \mathrm{MHz}, \mathrm{CDCl}_{3}\right): \delta 151.2,149.1,140.1,140.0,138.1,131.4$, 130.9, 130.4, 129.4, 128.4, 127.6, 127.5, 127.3, 125.4, 125.3, 125.0, 124.5, 123.2, 122.4, 122.0, 119.8 (aromatic C), 55.3 (spiro-C), 40.4 (alkyl-C), $35.2\left(\mathrm{Ar}-\mathrm{C}\left(\mathrm{CH}_{3}\right)_{3}\right), 31.9\left(\mathrm{Ar}-\mathrm{C}\left(\mathrm{CH}_{3}\right)_{3}\right), 30.1,29.7,29.4$, 29.3, 24.2, 22.7, 14.2 (alkyl-C) ppm; FT-IR (KBr): 3050, 2953, 2925, 2853, 1608, 1481, 1457, 1310 $\mathrm{cm}^{-1}$; mp 206-208 ${ }^{\circ} \mathrm{C}$; HR-MS $\left(\mathrm{FAB}^{+}\right.$) calcd. for $\mathrm{C}_{69} \mathrm{H}_{74}$ [M] 902.5791, found $\left[\mathrm{M}^{+}\right]$902.5786.

\section{Acknowledgements}

We thank JFE Chemical for the kind gift of some fluorene compounds. This work was supported by the Industrial Technology Research and Development Grant (09C46622) from NEDO of Japan.

\section{Appendix}

${ }^{1} \mathrm{H}-\mathrm{NMR}$ and ${ }^{13} \mathrm{C}$ NMR spectra of $\mathbf{3}$ and $\mathbf{6}$

Figure S1. ${ }^{1} \mathrm{H}-\mathrm{NMR}$ spectrum of 2,2' $7,7^{\prime}$-tetrakis(7-tert-butyl-1-pyrenyl)-9,9'-spirobi [9Hfluorene] (4-PySBF, $400 \mathrm{MHz}, \mathrm{THF}-\mathrm{d}_{8}$ ).

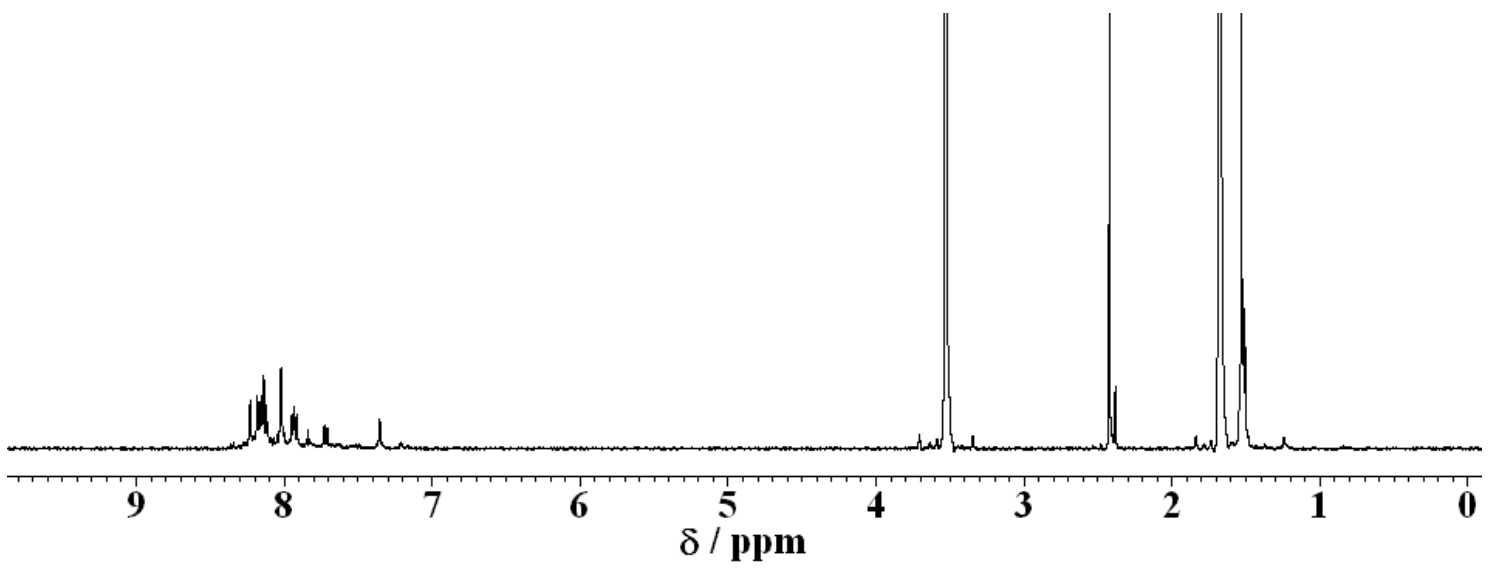


Figure S2. ${ }^{13} \mathrm{C}-\mathrm{NMR}$ spectrum of 2,2',7,7'-tetrakis(7-tert-butyl-1-pyrenyl)-9,9'-spirobi [9H-fluorene] (4-PySBF, $100 \mathrm{MHz}, \mathrm{CDCl}_{3}$ ).

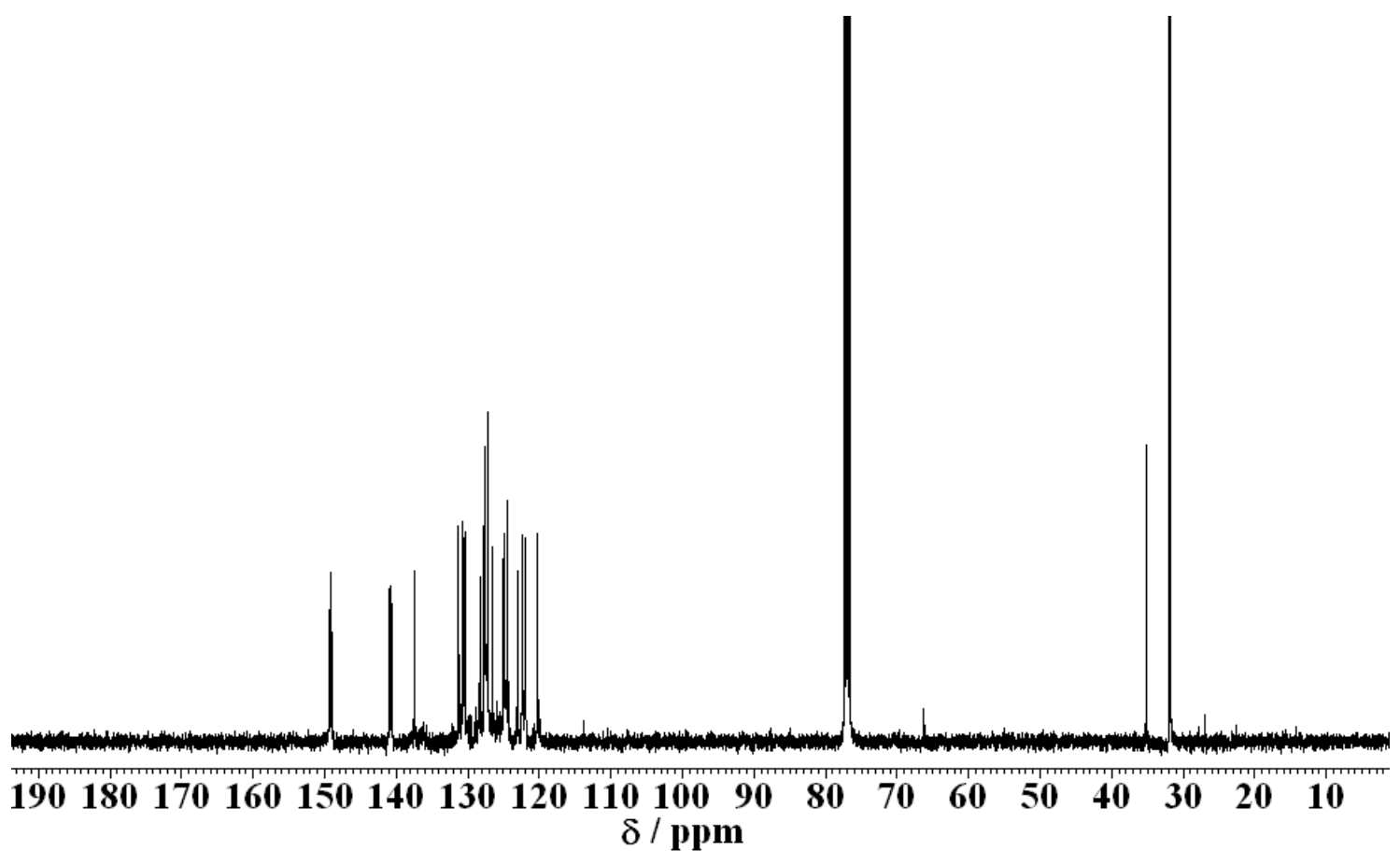

Figure S3. ${ }^{1} \mathrm{H}-\mathrm{NMR}$ spectrum of 9,9'-dioctyl-2,7-bis (7-tert-butyl-1-pyrenyl)-9H-fluorene (2-PyF, $400 \mathrm{MHz}, \mathrm{CDCl}_{3}$ ).

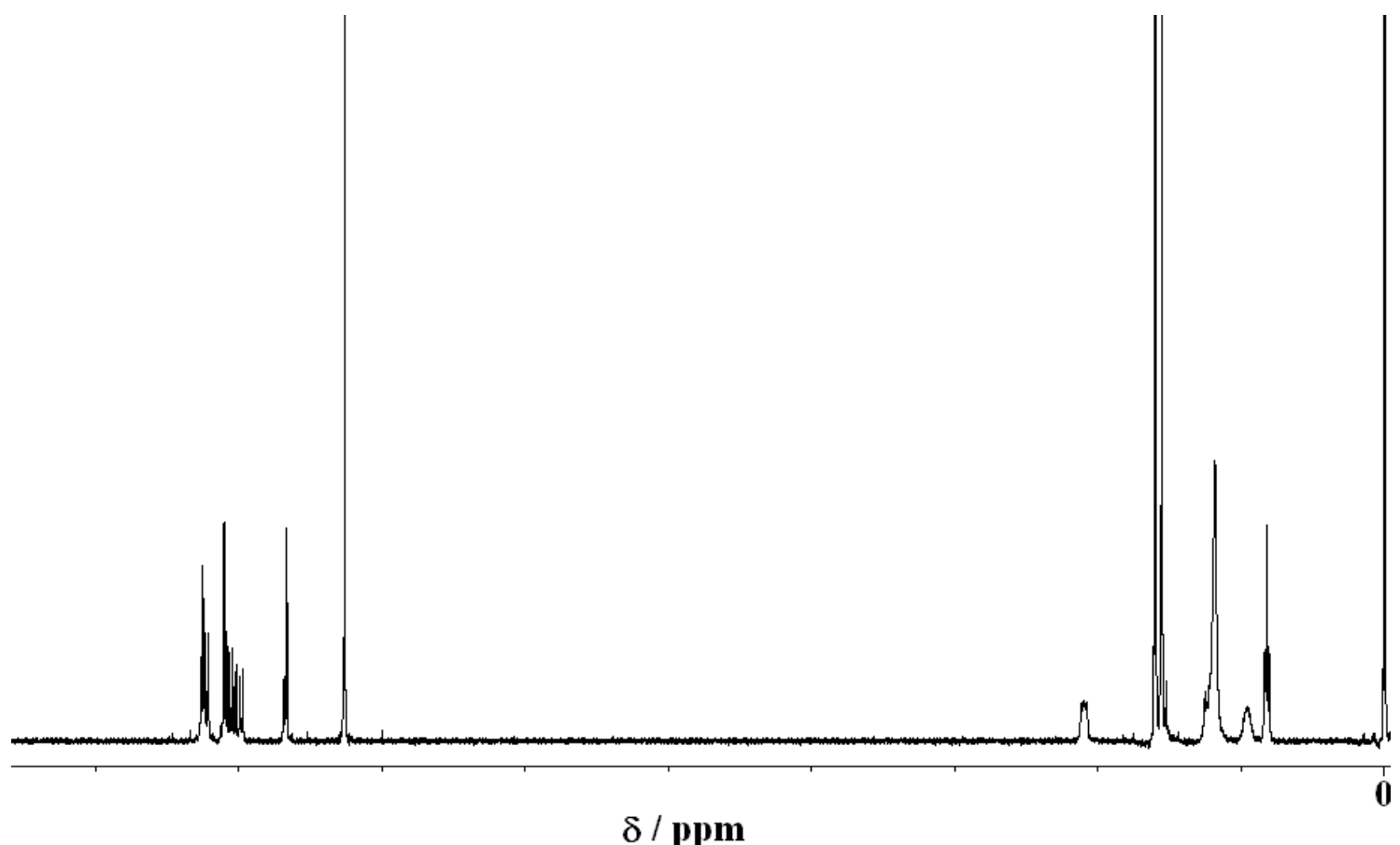


Figure S4. ${ }^{13} \mathrm{C}$-NMR spectrum of 9,9'-dioctyl-2,7-bis (7-tert-butyl-1-pyrenyl)-9H-fluorene (2-PyF, $100 \mathrm{MHz}, \mathrm{CDCl}_{3}$ ).

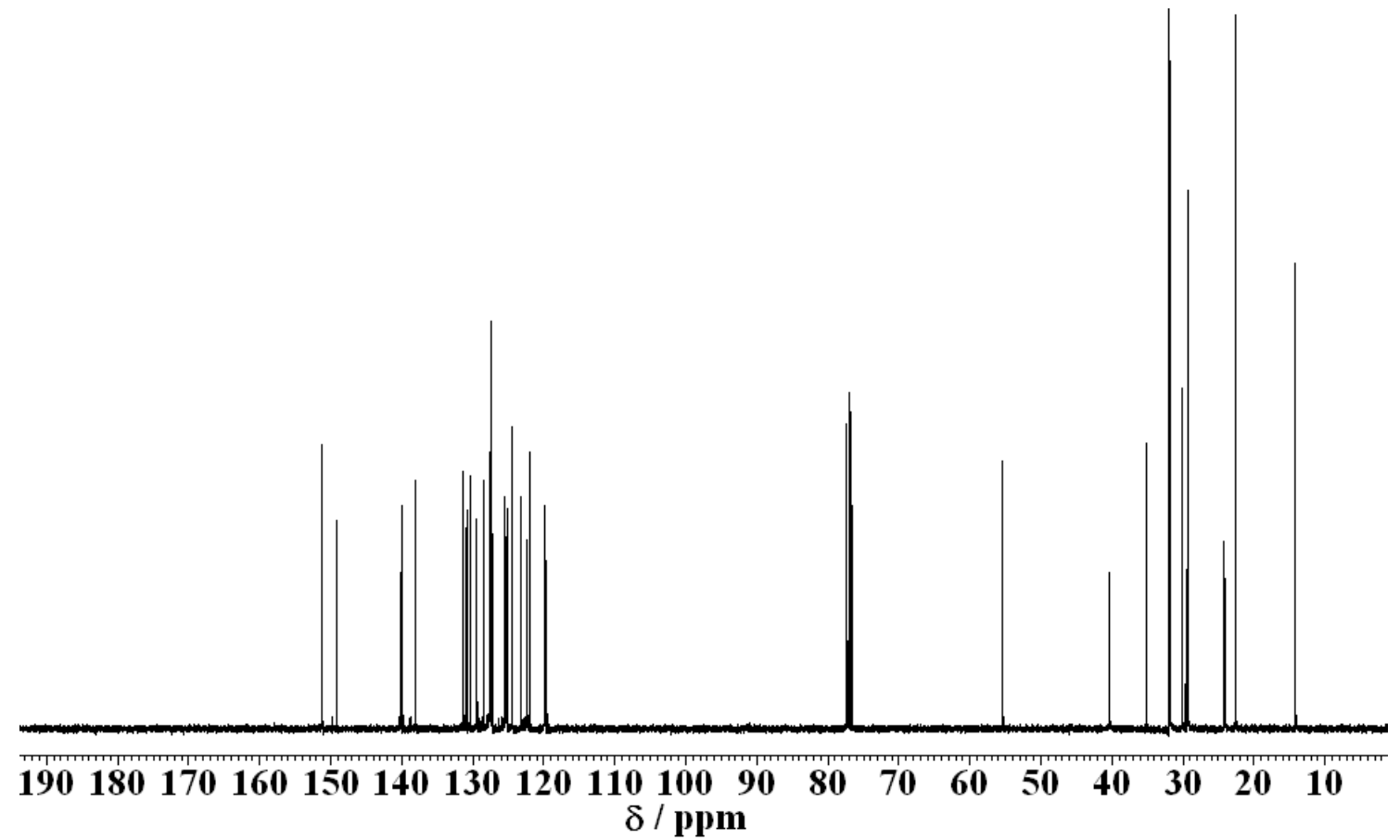

\section{References and Notes}

1. Yamaguchi, Y.; Tanaka, T.; Kobayashi, S.; Wakamiya, T.; Matsubara, Y.; Yoshida, Z. LightEmitting Efficiency Tuning of Rod-Shaped $\pi$-Conjugated Systems by Donor and Acceptor Groups. J. Am. Chem. Soc. 2005, 127, 9332-9333.

2. Yamaguchi, Y.; Ochi, T.; Wakamiya, T.; Matsubara, Y.; Yoshida, Z. New Fluorophores with Rod-Shaped Polycyano $\pi$-Conjugated Structures: Synthesis and Photophysical Properties. Org. Lett. 2006, 8. 717-720.

3. Yamaguchi, Y.; Matsubara, Y.; Ochi, T.; Wakamiya, T.; Yoshida, Z. How the $\pi$-Conjugation Length Affects the Fluorescence Emission Efficiency. J. Am. Chem. Soc. 2008, 130, 13867-13869.

4. Kanibolotsky, A.L.; Berridge, R.; Skabara, P.J.; Perepichka, I.F.; Bradley, D.D.C.; Koeberg, M. Synthesis and Properties of Monodisperse Oligofluorene-Functionalized Truxenes: Highly Fluorescent Star-Shaped Architectures. J. Am. Chem. Soc. 2004, 126, 13695-13702.

5. Leventis, N.; Rawashdeh, A.M.M.; Elder, I.A.; Yang, J.; Dass, A.; Sotiriou-Leventis, C. Synthesis and Characterization of $\mathrm{Ru}(\mathrm{II})$ Tris(1,10-phenanthroline)-Electron Acceptor Dyads Incorporating the 4-Benzoyl- $N$-methylpyridinium Cation or $N$-Benzyl- $N N^{6}$-methyl Viologen. Improving the Dynamic Range, Sensitivity, and Response Time of Sol-Gel-Based Optical Oxygen Sensors. Chem. Mater. 2004, 16, 1493-1506.

6. Barltrop, J.A.; Coyle, J.D. Principles of Photochemistry; Wiley: New York, NY, USA, 1987; p. 68.

7. Sagari, T.P.I.; Spehr, T.; Siebert, A.; Fuhmann-Lieker, T.; Salbeck, J. Spiro Compounds for Organic Optoelectronics. Chem. Rev. 2007, 107, 1011-1065. 
8. Cocherel, N.; Poriel, C.; Vignau, L.; Bergamini, J.; Rault-Berthelot, J. A New Blue Emitter for Nondoped Organic Light Emitting Diode Applications. Org. Lett. 2010, 12, 452-455.

9. Thirion, D.; Poriel, C.; Barriere, F.; Metivier, R.; Jeannin, O.; Rault-Berthelot, J. Tuning the Optical Properties of Aryl-Substituted Dispirofluorene-indenofluorene Isomers through Intramolecular Excimer formation. Org. Lett. 2009, 11, 4794-4797.

10. Luo, J.; Zhou, Y.; Niu, Z.Q.; Zhou, Q.F.; Ma, Y.; Pei, J. Three-Dimensional Architectures for Highly Stable Pure Blue Emission. J. Am. Chem. Soc. 2007, 129, 113414-113415.

11. Pudzich, R.; Fuhrmann-Lieker, T.; Salbeck, J. Spiro Compounds for Organic Electroluminescence and Related Applications. Adv. Polym. Sci. 2006, 199, 83-142.

12. Kim, S.Y.; Lee, M.; Boo, B.H. Second molecular hyperpolarizability of 2,2'-diamino-7,7'-dinitro9,9'-spirobifluorene: An experimental study on third-order nonlinear optical properties of a spiroconjugated dimer. J. Chem. Phys. 1998, 109, 2593-2595.

13. Poriel, C.; Ferrand, Y.; Maux, P.L.; Rault-Berthelot, J.; Simonneaux, G. Organic Cross-Linked Electropolymers as Supported Oxidation Catalysts: Poly((tetrakis(9,9`spirobifluorenyl)porphyrin) -manganese) Films. Inorg. Chem. 2004, 43, 5086-5095.

14. Poriel, C.; Ferrand, Y.; Maux, P.L.; Paul, C.; Rault-Berthelot, J.; Simonneaux, G. Poly(ruthenium carbonyl spirobifluorenylporphyrin): A new polymer used as a catalytic device for carbene transfer. Chem. Commun. 2003, 2308-2309.

15. Wong, K.T.; Ku, S.Y.; Cheng, Y.M.; Lin, X.Y.; Hung, Y.Y.; Pu, S.C.; Chou, P.T.; Lee, G.H.; Peng, S.M. Synthesis, Structures, and Photoinduced Electron Transfer Reaction in the 9,9'Spirobifluorene-Bridged Bipolar Systems. J. Org. Chem. 2006, 71, 456-465.

16. Chien, Y.Y.; Wong, K.T.; Chou, P.T.; Cheng, Y.M. Syntheses and spectroscopic studies of spirobifluorene-bridged bipolar systems; photoinduced electron transfer reactions. Chem. Commun. 2002, 2874-2875.

17. Sartin, M.M.; Shu, C.; Bard, A.J. Electrogenerated Chemiluminescence of a SpirobifluoreneLinked Bisanthracene: A Possible Simultaneous, Two-Electron Transfer. J. Am. Chem. Soc. 2008, 130, 5354-5360.

18. Kowada, T.; Matsuyama, Y.; Ohe, K. Synthesis, Characterization, and Photoluminescence of Thiophene-Containing Spiro Compounds. Synlett 2008, 12, 1902-1906.

19. Seto, R.; Sato, T.; Kojima, T.; Hosokawa, K.; Koyama, Y.; Takata, T. 9,9'-SpirobifluoreneContaining Polycarbonates: Transparent Polymers with High Refractive Index and Low Birefringence. J. Polym. Sci. Part A Polym. Chem. 2010, 48, 3658-3667.

20. Lei, T.; Luo, J.; Wang, L.; Ma, Y.; Wang, J.; Cao, Y.; Pei, J. Highly stable blue light-emitting materials with a three-dimensional architecture: Improvement of charge injection and electroluminescence performance. New J. Chem. 2010, 34, 699-707.

21. Liao, Y.L., Lin, C.Y.; Wong, K.T.; Hou, T.H.; Hung, W.Y. A Novel Ambipolar Spirobifluorene Derivative that Behaves as an Efficient Blue-Light Emitter in Organic Light-Emitting Diodes. Org. Lett. 2007, 9, 4511-4514.

22. Cocherel, N.; Poriel, C.; Rault-Berthelot, J.; Barriere, F.; Audebrand, N.; Slawin, A.M.Z.; Vignau, L. New 3 $\pi$-2Spiro Ladder-Type Phenylene Materials: Synthesis, Physicochemical Properties and Applications in OLEDs. Chem. Eur. J. 2008, 14, 11328-11342. 
23. Shimizu, H.; Fujimoto, K.; Furusho, M.; Maeda, H.; Nanai, Y.; Mizuno, K.; Inouye, M. Highly Emissive -Conjugated Alkynylpyrene Oligomers: Their Synthesis and Photophysical Properties. J. Org. Chem. 2007, 72, 1530-1533.

24. Oyamada, T.; Akiyama, S.; Yahiro, M.; Saigou, M.; Shiro, M.; Sasabe, H.; Adachi, C. Unusual photoluminescence characteristics of tetraphenylpyrene (TPPy) in various aggregated morphologies. Chem. Phys. Lett. 2006, 421, 295-299.

25. Uchimura, M.; Watanabe, Y.; Araoka, F.; Watanabe, J.; Takezoe, H.; Konishi, G. Development of Laser Dyes to Realize Low Threshold in Dye-doped Cholesteric Liquid Crystal Lasers. Adv. Mater. 2010, 22, 4473-4478.

26. Watanabe, Y.; Uchimura, M.; Araoka, F.; Konishi, G.; Watanabe, J.; Takezoe, H. Extremely Low Threshold in a Pyrene-doped Distributed Feedback Cholesteric Liquid Crystal Laser. Appl. Phys. Express 2009, 2, 102501.

27. Figueira-Duarte, T.M.; Simon, S.C.; Wagner, M.; Druzhinin, S.I.; Zachariasse, K.A.; Müllen, K. Polypyrene Dendrimers. Angew. Chem. Int. Ed. 2008, 47, 10175-10178.

28. Schweig, A.; Weidner, U.; Hellwinkel, D.; Krapp, W. Spiroconjugation. Angew. Chem. Int. Ed. 1973, 12, 310-311.

29. Cocherel, N.; Poriel, C.; Jeannin, O.; Yassin, A.; Rault-Berthelot, J. The synthesis, physicochemical properties and anodic polymerization of a novel ladder pentaphenylene. Dyes Pigm. 2009, 83, 339-347.

30. Poriel, C.; Liang, J.J.; Rault-Berthelot, J.; Barriere, F.; Cocherel, N.; Slawin, A.M.Z.; Horhant, D.; Virboul, M.; Alcaraz, G.; Audebrand, N.; Vignau, L.; Huby, N.; Wantz, G.; Hirsch. L. Dispirofluorene-Indenofluorene Derivatives as New Building Blocks for Blue Organic Electroluminesent Devices and Electroactive Polymers. Chem. Eur. J. 2007, 13, 10055-10069.

31. Tao, S.; Peng, Z.; Zhang, X.; Wang, P.; Lee, C.S.; Lee, S.T. Highly Efficient Non-Doped Blue Organic Light-Emitting Diodes Based on Fluorene Derivatives with High Thermal Stability. Adv. Funct. Mater. 2005, 15, 1716-1721.

32. Johansson, N.; dos Santos, D.A.; Guo, S.; Cornil, J.; Fahlman, M.; Salbeck, J.; Schenk, H.; Arwin, H.; Bredas, J.L.; Salanek, W.R. Electronic Structure and Optical Properties of Electroluminescent Spiro-type Molecules. J. Chem. Phys. 1997, 107, 2542-2549.

33. This phenomenon is thought to be caused by intramolecular overlapping of the electron clouds of the 2,7-dipyrenylfluorene units. Few researchers have successfully observed distinct and strong spiroconjugation in the excited state [29,30]. However, in general, observed spiroconjugations were weak [31]. For example, in ref. [31], the authors reported that a compound in which two pyrenes were introduced into the spirobifluorene skeleton (2,7-dipyrene-9,9'-spirobifluorene, SDPF) showed a red-shift of $4 \mathrm{~nm}$ (in the ground state) and $1 \mathrm{~nm}$ (in the excited state) in comparison with a model compound, 2,7-dipyrene-9,9'-dimethyl fluorene (DPF). Futhermore in ref. [32], the authors compared UV-Vis spectra between a spiro-compound and an appropriate model compound by using MO calculations. However, to the best of our knowledge, no comparison between a 2,7-diarylfluorene and an appropriate spiro-compound (2,2',7,7'tetraarylspirobifluorene) has been carried out yet to investigate the spiroconjugation by experimental methods. This pyrene-fluorene-conjugated compound (4-PySBF) exhibited extremely large red-shift of fluorescence $(20 \mathrm{~nm})$. This phenomenon is very interesting. We 
intend to calculate the electron states of these compounds in the ground and excited states by the MO method and discuss this conjugation system in greater detail.

34. Maeda, H.; Maeda, T.; Mizuno, K.; Fujimoto, K.; Shimizu, H.; Inouye, M. Alkynylpyrenes as Improved Pyrene-Based Biomolecular Probes with the Advantages of High Fluorescence Quantum Yields and Long Absorption/Emission Wavelengths. Chem. Eur. J. 2006, 12, 824-831.

35. Abdel-Shafi, A.A.; Wilkinson, F. Charge Transfer Effects on the Efficiency of Singlet Oxygen Production Following Oxygen Quenching of Excited Singlet and Triplet States of Aromatic Hydrocarbons in Acetonitrile. J. Phys. Chem. A. 2000, 104, 5747-5757.

36. Kyushin, S.; Ikarugi, M.; Goto, M.; Hiratsuka, H.; Matsumoto, H. Synthesis and Electronic Properties of 9,10-Disilylanthracenes. Organometallics 1996, 15, 1067-1070.

37. Usui, Y.; Shimizu, N.; Mori, S. Mechanism on the Efficient Formation of Singlet Oxygen by Energy Transfer from Excited Singlet and Triplet States of Aromatic Hydrocarbons. Bull. Chem. Soc. Jpn. 1992, 65, 897-902.

38. McLean, A.J.; McGarvey, D.J.; Truscott, T.G.; Lambert, C.R.; Land, E.J. Effect of Oxygenenhanced Intersystem Crossing on the observartion efficiency of Formation of Singlet Oxygen. $J$. Chem. Soc. Faraday Trans. 1990, 86, 3075-3080.

39. Pyrene has a $k_{\text {isc }}$ value of $1.2 \times 10^{6} \mathrm{~S}^{-1}$ in cyclohexane, see Kikuchi, K. A new method for determining the efficiency of enhanced intersystem crossing in fluorescence quenching by molecular oxygen. Chem. Phys. Lett. 1991, 183, 103-106.

Sample Availability: Available from GK.

(C) 2010 by the authors; licensee MDPI, Basel, Switzerland. This article is an open access article distributed under the terms and conditions of the Creative Commons Attribution license (http://creativecommons.org/licenses/by/3.0/). 